\title{
Can prematurity risk in twin pregnancies after in vitro fertilization be predicted? A retrospective study Andrea Weghofer*1,2, Katharina Klein ${ }^{1}$, Maria Stammler-Safar1, Christof Worda1 ${ }^{1}$, David H Barad ${ }^{2}$, Peter Husslein ${ }^{1}$ and Norbert Gleicher ${ }^{2,3}$
}

\author{
Address: ${ }^{1}$ Department of Obstetrics \& Gynecology, Medical University Vienna, Vienna, Austria, ${ }^{2}$ The Center for Human Reproduction and The \\ Foundation for Reproductive Medicine, New York, New York, USA and ${ }^{3}$ Department of Obstetrics, Gynecology and Reproductive Sciences, Yale \\ Universiy School of Medicine, New Haven, Connecticut, USA \\ Email: Andrea Weghofer* - andrea.weghofer@meduniwien.ac.at; Katharina Klein - katharina.klein@meduniwien.ac.at; Maria Stammler- \\ Safar - maria.stammler-safar@meduniwien.ac.at; Christof Worda - christof.worda@meduniwien.ac.at; David H Barad - dbarad@thechr.com; \\ Peter Husslein - peter.husslein@meduniwien.ac.at; Norbert Gleicher - ngleicher@thechr.com \\ * Corresponding author
}

Published: 25 November 2009

Reproductive Biology and Endocrinology 2009, 7:136 doi:10.1186/1477-7827-7-136

This article is available from: http://www.rbej.com/content/7/I//36

(C) 2009 Weghofer et al; licensee BioMed Central Ltd.

This is an Open Access article distributed under the terms of the Creative Commons Attribution License (http://creativecommons.org/licenses/by/2.0), which permits unrestricted use, distribution, and reproduction in any medium, provided the original work is properly cited.
Received: 6 October 2009

Accepted: 25 November 2009

\begin{abstract}
Background: Assisted reproduction (ART) contributes to world-wide increases of twin pregnancies, in turn raising prematurity risks. Whether characteristics of ART cycles, resulting in twin gestations, can predict prematurity risks was the subject of this study.

Methods: One-hundred-and-six women, ages 20 to 39 years, with consecutive dichorionicdiamniotic (DC/DA) twin gestations were retrospectively investigated. All pregnancies investigated followed fresh ART cycles, with use of autologous gamets, and were delivered at a university-based high-risk, maternal-fetal medicine unit. Only premature deliveries (i.e., <37.0 weeks gestational age), with viable neonate(s) of $\geq 500$ grams, were considered for analysis.

Results: After I.8 +/- I.2 ART cycles, I I.0 +/- 5.4 oocytes were retrieved and $2.4+/-0.9$ embryos transferred in 106 women aged $31.6+/-4.2$ years. Indications for ART treatment were male factor in $51.9 \%$, female infertility in $27.4 \%$ and combined infertility in $20.8 \%$. Though maternal age significantly influenced prematurity risk $(p<0.05)$, paternal age, maternal body mass index, indications for fertility treatment, number of previous ART attempts, oocytes retrieved or embryos transferred, as well as stimulation protocols and previous ART pregnancies, were not associated with gestational duration in twin pregnancies.
\end{abstract}

Summary: Except for female age, baseline and ART cycle characteristics do not allow for prediction of prematurity risk in dichorionic twin gestations after assisted reproduction.

\section{Background}

Since Louise Brown, the first in vitro fertilization (IVF) birth in 1978, more than three million IVF children have been born [1]. Assisted reproduction (ART), nevertheless, has come under criticism due to the increased risk of multiple births associated with this fertility treatments [2]. Twin pregnancies after spontaneous conception occur in Caucasians at a rate of $1: 80$. Assisted reproduction in 
Europe, in contrast, currently results in twin rates of $21.7 \%$ with wide variations across the countries [3]. Twins (and higher order multiples), in comparison to singleton gestations, are at increased risk for prematurity-associated adverse perinatal outcome $[4,5]$. A broader application of single embryo transfer (SET) has been suggested to reduce this rising incidence of multiple pregnancies in the course of assisted reproduction without compromising pregnancy chances $[6,7]$.

Bechoua et al., for instance, describe comparable pregnancy chances after double embryo transfer (DET) when compared to elective single embryo transfer in a cohort of good prognosis patients [8]. A Finnish group even report lower costs and higher cumulative live birth rates in good prognosis patients after SET and cryo-embryo transfers compared to DET $[9,10]$. Others, however, claim that SET may after all reduce pregnancy chances. Veleva et al., for instance, differentiate between elective and compulsory SET. In their retrospective analysis, they describe considerably lower pregnancy rates in women undergoing SET of a non top quality embryo or compulsory SET in comparison to double embryo transfers or elective SETs of a top quality embryo [11]. Roberts et al. go even further - they report a need for a 55\% rate of SET to reduce twin rates to ten percent. By doing so, pregnancy rates would drop by $19 \%$ [12]. In a recent meta-analysis, Gelbaya et al. are in accordance with these data. They report a statistically significant reduction in the probability of live birth $(-38 \%)$ and multiple birth (-94\%) after e-SET [13].

The ability to prospectively identify IVF patients at increased risk towards twinning and premature delivery, would, therefore, allow for a more selective utilization of SET and therefore, possibly, more effectively contribute to the reduction of adverse perinatal outcome in IVF twins [12]. Identifying such a subset of patients at risk, would then more than compensate for potentially impaired pregnancy chances in women who are not good prognosis candidates for SET.

While a variety of prenatal risk factors for preterm births in twin gestations have been established [14], conflicting data exist on the potential impact of controlled ovarian hyperstimulation on prematurity risk and perinatal outcome. Griesinger and colleagues, for instance, fail to find an impact of controlled ovarian hyperstimulation on birth weight [15], while Shih et al. report lower birth weight in singletons after fresh embryo transfer when compared to frozen cryopreservation cycles [16]. Abramov et al. go even further, showing higher rates of prematurity, low birth weight, pregnancy-induced hypertension and placental abruption in pregnancies after ovarian hyperstimulation syndrome (OHSS) [17]. Aytoz concur with these observations, describing increased rates of intrauterine deaths in ART pregnancies with severe male factor infertility [18].

These publications point toward a potentially negative influence of IVF cycle characteristics, such as male factor infertility [18] and good response to controlled ovarian hyperstimulation or OHSS [17], on pregnancy outcome and prematurity risk. Male factor infertility and OHSS are, however, considered risk factors for the occurrence of multiple gestations $[12,19]$.

Whether other ART cycle characteristics, such as indication for fertility treatment and ovarian response to stimulation, serve as predictive for severe prematurity in twin gestations after ART was the subject of the here presented study.

\section{Discussion \\ Methods}

The present retrospective study involved 106 women, aged 20 to 39 years, with dichorionic-diamniotic (DC/ DA) twin gestations after in vitro fertilization (IVF). All pregnancies were established with autologous oocytes/ sperm in fresh IVF cycles and via the transfer of two or more embryos. To ensure IVF-related conception and a complete history of IVF-related parameters, such as the number of embryos transferred, only IVF-Fonds-covered pregnancies were included. [The IVF-Fonds provides governmental financial support of up to four IVF cycles per pregnancy in women under age 40 [20]]. To determine chorionicity, patients underwent first trimester ultrasound scans, performed by a small group of experienced, specifically and uniformly trained senior physicians. The detection of lambda signs served as proof of dichorionicity.

All women underwent prenatal care and delivery at the Department of Obstetrics and Gynecology at the Medical University Vienna, a University-based hospital unit for highrisk maternal-fetal medicine. To exclude potential prematurity-associated biases, neither monochorionic twin gestations, nor pregnancies that had undergone selective fetal reduction or pregnancies with vanishing embryos were eligible for enrollment. After 37 weeks of gestation, a number of twin pregnancies were delivered electively. To exclude this potential bias, only twin gestations that were delivered preterm (i.e., gestational age $<37.0$ weeks) were included in the study. A comparison of IVF cycle characteristics between study patients and DC twin gestations that delivered at term (i.e., $\geq 37$ weeks; not eligible for enrollment) revealed no significant differences between the groups.

Statistical influences of maternal and paternal ages, numbers of previous IVF attempts and pregnancies, indica- 
tions for fertility treatment, stimulation protocols, oocyte yields, fertilization procedure (IVF or ICSI), number of embryos fertilised/transferred, smoking status and body mass index on gestational ages were investigated. The data analyses of pregnancy- and delivery-related maternal and neonatal outcome data were based on retrospective chart reviews and computer-generated databases at the Department of Obstetrics and Gynecology at the Medical University Vienna. Assisted reproduction technology (ART)-related data were collected by chart review and, where applicable, from of a computer-generated database at the IVF-Fonds.

\section{Statistics}

Statistical analyses were performed utilizing SPSS version 10.0. Quantitative variables are summarized by their mean (standard deviation), while qualitative variables are summarized by frequency tables. Univariate and multivariate analysis were performed by (stepwise) linear regression. Pearson's Correlation was used to determine the sample size required to detect a correlation between gestational duration and ART cycle characteristics. In order to detect a correlation of 0.25 with a power of $80 \%$ and a Type I error of 5\%, a sample size of 98 women was needed. Patient characteristics known to influence prematurity risk in spontaneous pregnancies (i.e., age, smoking status, body mass index, parity) [14] were investigated in univariate analysis and included in multivariate analysis if significant (i.e., p < 0.05). ART cycle characteristics (i.e., numbers of previous IVF attempts and pregnancies, indications for fertility treatment, stimulation protocols, oocyte yields, fertilization procedure (IVF or ICSI), number of embryos fertilized/transferred) were also investigated in univariate regression analysis. While number of previous IVF-attempts, stimulation protocols, fertilization procedure (IVF or ICSI) and number of embryos transferred were only included in multi-regression analysis if significant in univariate analysis, factors previously described to be associated with prematurity risk, such as maternal age, number of previous IVF-pregnancies, indication for fertility treatment and oocyte yield $[14,17,18]$, were included in multivariate regression analyses, irrespective of their statistical significance in univariate analysis. Univariate and multivariate regression analyses were then performed in the same fashion for women $\leq 30$ years and $>30$ years, except for the exclusion of maternal age.

A comparison of ART cycle characteristics between study patients and DC twin gestations that delivered at term (i.e., $\geq 37$ weeks; not eligible for enrollment) revealed no significant differences between the groups. P-values of < 0.05 were considered statistically significant. Institutional Review Board (IRB) approval for data-linkage and retrospective data analyses was obtained from the IRB at the Medical University Vienna.

\section{Results}

Women demonstrated a mean age of $31.6+/-4.2$ years and a mean body mass index of $23.7+/-4.34 \mathrm{~kg} / \mathrm{m}^{2}$. Men presented with a mean age of $33.9+/-4.5$ years. Indications for in vitro fertilization treatment were male factor in $51.6 \%$, female infertility in $27.4 \%$ and combined infertility in $20.8 \%$. Women with female infertility demonstrated with tubal infertility in $64.7 \%, 23.5 \%$ were patients with polycystic ovary syndrome (PCOS), 9.8\% suffered from endometriosis and $2 \%$ had a history of tubal ligation. In addition to PCOS, one patient demonstrated with infertility-associated immunological factors. Due to the present distribution of fertility indications, intracytoplasmatic sperm injection (ICSI) was required in $68.9 \%$ of cases.

To achieve their dichorionic-diamniotic pregnancies, couples underwent a mean number of 1.8+/- 1.2 ART cycles. More than two ART attempts were required in $21.7 \%$ of patients. IVF attempts with concurrent twin pregnancy demonstrated a mean number of $11.0+/-5.4$ oocytes retrieved and $6.6+/-3.5$ oocytes fertilised. A mean number of $2.4+/-0.9$ embryos were transferred. No statistically significant difference was observed in the number of embryos transferred according to cycle number and previous IVF outcome (first IVF attempts, $2.3+$ /- 0.7; previous IVF failure, $2.7+/-1.1$; previous IVF pregnancies, 2.4 +/- 0.9 embryos). The couples' ages, their indications for fertility treatments, oocyte yields, fertilization rates and other IVF/ICSI cycle characteristics were also comparable between first ART attempts, previous ART failures and previous ART pregnancies.

Delivery occurred at a mean gestational age of $33.6+/-2.9$ weeks and resulted in 105 female and 107 male neonates. The mean birth weight for both twins was $2011.3+$ /$527.9 \mathrm{gm}$ (Twin A) and 1927.9 +/- $549.2 \mathrm{gm}$ (Twin B), respectively, with a mean discordance of $9.6+/-13.4$ percent. Mean perinatal arterial $\mathrm{pH}$ for both twins was 7.26 +/- 0.1 (Twin A) and 7.26 +/- 0.1 (Twin B). Delivery resulted in $99.1 \%$ live births for Twin A and B. Immediately post partum, $46.2 \%$ of first twins and $50.0 \%$ of second twins required neonatal intensive care at the neonatal intensive care unit (NICU) of the Department of Pediatrics at the Medical University Vienna.

When the impact of maternal and paternal age, maternal body mass index and tobacco usage, previous pregnancies and IVF attempts, stimulation (GnRH agonist or GnRH antagonist protocol), oocyte yield, fertilization rate and number of embryos transferred on gestational age were calculated in univariate analysis, only maternal age reached statistical significance $(\mathrm{p}<0.05)$. In multi-regression analyses, including maternal age, oocyte yield and 
number of previous IVF-pregnancies, only maternal age demonstrated to be predictive for gestational duration.

When univariate and multivariate regression were performed according to age groups (i.e., $\leq 30$ years and $>30$ years for female age), maternal age did not reach significance in univariate analysis and was, therefore, not included in multi-regression. When multi-regression analyses, including number of oocytes retrieved, number of previous IVF-pregnancies and indication for fertility treatment, were then performed for those age groups (i.e., $\leq 30$ years and $>30$ years), none of those characteristics included proved statistically associated with prematurity risk in twin gestations.

Many improvements in perinatal and neonatal medicine have led to increased survival rates in premature neonates. Their neurodevelopmental outcomes, particularly of those with very low birth weight and/or very early gestational age, are, however, still concerning and often followed by a variety of lifelong disabilities [21]. Assisted reproduction represents such a risk factor for prematurity in both, singletons and multiple pregnancies [22].

The uniformly agreed to goal of ART is the safest possible delivery of greatly desired offspring. SET, in its current form, focuses, however, on prevention of twin pregnancies, rather than on reduction of risks, associated with twin pregnancies. By doing so, single embryo transfer is mainly performed in good prognosis, i.e. young, IVF patients [23]. Obstetrical data, however, demonstrate an exponentially increasing prematurity risk with advancing maternal age [24]. If we could prospectively identify a subset of women at increased risks after twin conception, following ART, those patients would especially benefit from single embryo transfer. Even women with impaired pregnancy chances after SET, such as older patients and those with diminished ovarian function $[25,26]$, would derive compensatory benefits to lower pregnancy chances by a possible reduction in prematurity risk.

Such risk-focused, rather than age-based, treatment policies have already been applied in prenatal diagnosis. While amniocentesis used to be a routine procedure in women above age 35 , this approach was abandoned in favor of an individual risk calculation [27]. This modification in approach increased diagnostic accuracy for fetal chromosomal abnormalities, but also saved a considerable number of prospective mothers of advanced age from the amniocentesis-related risks of a preterm premature rupture of membranes or miscarriage.

Our, unfortunately, negative findings in this study, may have a variety of explanations: (i) All women that were eligible for enrolment underwent close prenatal surveillance and delivery at a university-based high-risk, maternal-fetal medicine unit. Whatever ART-associated risk divergence may have been present, excellent prenatal care could have evened it out. (ii) This is further supported by IVF twins presenting with significantly lower perinatal mortality than spontaneously conceived twins $[4,28]$. (iii) Women in this study also do not necessarily reflect a typical IVF population. Our study patients represented IVF-patients under age 40 with normal body mass index; they were either themselves fertile (male factor) or suffered from tubal infertility, and had only a limited numbers of previous IVF attempts.

This stands in contrast to other indications for fertility treatments, which are statistically highly associated with impaired pregnancy outcome, such as autoimmunity, uterine abnormalities or a history of repeated pregnancy loss [29,30]. Moreover, our study population involved only fresh, autologous IVF cycles, reported associated with higher implantation potential and, consequently, higher risk for twin gestations, than cycles in which thawed embryos are used [31].

\section{Summary}

Standard ART cycle characteristics did not serve as predictive factors for severity of prematurity risk in our cohort of prematurely delivered dichorionic twin gestations after assisted reproduction. Since our study population consisted of a limited number of good prognosis patients, our results do not preclude that further research in more typical average IVF populations, at larger risk for prematurity, may after all, be able to define such risk factors. Further research is, therefore, warranted to develop a risk-focused approach to prevent, or at least reduce, adverse perinatal outcomes in twin pregnancies after ART.

\section{Competing interests}

The authors declare that they have no competing interests.

\section{Authors' contributions}

AW Substantial contributions to conception and design, acquisition, analysis and interpretation of data, drafting the article, final approval of the version to be published. KK and MSS Substantial contributions to acquisition and contributions to analysis of data, revising the article critically for important intellectual content, final approval of the version to be published. DHB, CW, PH Substantial contributions to conception of the study, analysis of data, revising the manuscript critically for important intellectual content, final approval of the version to be published. NG Substantial contributions to conception and design, analysis and interpretation of data, revising the manuscript critically for important intellectual content, and final approval of the version to be published. 


\section{Acknowledgements}

We are indebted to Dr. Renate Fally-Kausek and Mag. Reinhard Kern for their invaluable assistance with data linkage.

\section{References}

I. Allen VM, Wilson RD, Cheung A: Pregnancy outcomes after assisted reproductive technology. J Obstet Gynaecol Can 2006, 28:220-250.

2. Liu $\mathrm{CH}$ : Impact of assisted reproductive technology on modern medicine. J Formos Med Assoc 2000, 99: 100-106.

3. Andersen AN, Goossens V, Ferraretti AP, Bhattacharya S, Felberbaum R, de Mouzon J, Nygren KG: Assisted reproductive technology in Europe, 2004: results generated from European registers by ESHRE. Hum Reprod 2008, 23:756-77I.

4. Helmerhorst FM, Perquin DA, Donker D, Keirse MJ: Perinatal outcome of singletons and twins after assisted conception: a systematic review of controlled studies. Bmj 2004, 328:26I.

5. Ombelet W, Martens G, De Sutter P, Gerris ], Bosmans E, Ruyssinck G, Defoort P, Molenberghs G, Gyselaers W: Perinatal outcome of I 2,02 I singleton and 3 I 08 twin births after non-IVF-assisted reproduction: a cohort study. Hum Reprod 2006, 2 I : I025-I032.

6. Pandian Z, Templeton A, Serour G, Bhattacharya S: Number of embryos for transfer after IVF and ICSI: a Cochrane review. Hum Reprod 2005, 20:268I-2687.

7. Leniaud L, Poncelet C, Porcher R, Martin-Pont B, Cedrin-Durnerin I, Hugues JN, Wolf JP, Sifer C: [Prospective evaluation of elective single-embryo transfer versus double-embryo transfer following in vitro fertilization: a two-year French hospital experience]. Gynecol Obstet Fertil 2008, 36:159-165.

8. Bechoua S, Astruc K, Thouvenot S, Girod S, Chiron A, Jimenez C, Sagot P: How to demonstrate that eSET does not compromise the likelihood of having a baby? Hum Reprod 2009, 24( I 2):3073-308I.

9. Moustafa MK, Sheded SA, El Aziz Mousta MA: Elective single embryo transfer versus double embryo transfer in assisted reproduction. Reprod Biomed Online 2008, I 7:82-87.

10. Veleva Z, Karinen P, Tomas C, Tapanainen JS, Martikainen H: Elective single embryo transfer with cryopreservation improves the outcome and diminishes the costs of IVF/ICSI. Hum Reprod 2009, 24:I632-1639.

II. Veleva Z, Vilska S, Hyden-Granskog C, Tiitinen A, Tapanainen JS, Martikainen $\mathrm{H}$ : Elective single embryo transfer in women aged 3639 years. Hum Reprod 2006, 21 :2098-2102.

12. Roberts SA, Fitzgerald CT, Brison DR: Modelling the impact of single embryo transfer in a national health service IVF programme. Hum Reprod 2009, 24:|22-|3|.

13. Gelbaya TA, Tsoumpou I, Nardo LG: The likelihood of live birth and multiple birth after single versus double embryo transfer at the cleavage stage: a systematic review and meta-analysis. Fertil Steril 2009 in press.

14. Klein K, Gregor H, Hirtenlehner-Ferber K, Stammler-Safar M, Witt A, Hanslik A, Husslein P, Krampl E: Prediction of spontaneous preterm delivery in twin pregnancies by cervical length at midgestation. Twin Res Hum Genet 2008, I I:552-557.

15. Griesinger G, Kolibianakis EM, Diedrich K, Ludwig M: Ovarian stimulation for IVF has no quantitative association with birthweight: a registry study. Hum Reprod 2008, 23:2549-2554.

16. Shih W, Rushford DD, Bourne H, Garrett C, McBain JC, Healy DL, Baker HW: Factors affecting low birthweight after assisted reproduction technology: difference between transfer of fresh and cryopreserved embryos suggests an adverse effect of oocyte collection. Hum Reprod 2008, 23: I644-I653.

17. Abramov Y, Elchalal U, Schenker JG: Obstetric outcome of in vitro fertilized pregnancies complicated by severe ovarian hyperstimulation syndrome: a multicenter study. Fertil Steril 1998, 70:1070-1076.

18. Aytoz A, Camus M, Tournaye H, Bonduelle M, Van Steirteghem A, Devroey $P$ : Outcome of pregnancies after intracytoplasmic sperm injection and the effect of sperm origin and quality on this outcome. Fertil Steril 1998, 70:500-505.

19. Cupisti S, Emran J, Mueller A, Dittrich R, Beckmann MW, Binder H: Course of ovarian hyperstimulation syndrome in 19 intact twin pregnancies after assisted reproduction techniques, with a case report of severe thromboembolism. Twin Res Hum Genet 2006, 9:691-696.
20. Bundeskanzleramt_Österreich: IVF-Fonds-Gesetz-Novelle 2004. Bundesgesetzblatt der Republik Österreich 2004. NR: GP XXII RV 369 AB 445 S. 56. BR: AB 7027:707

21. Moddemann D, Shea S: The developmental paediatrician and neonatal follow-up. Paediatr Child Health 2006, I I:295.

22. Allen C, Bowdin S, Harrison RF, Sutcliffe AG, Brueton L, Kirby G, Kirkman-Brown J, Barrett C, Reardon W, Maher E: Pregnancy and perinatal outcomes after assisted reproduction: a comparative study. Ir J Med Sci 2008, I77:233-24I.

23. Stillman RJ, Richter KS, Banks NK, Graham JR: Elective single embryo transfer: A 6-year progressive implementation of 784 single blastocyst transfers and the influence of payment method on patient choice. Fertil Steril 2008 in press.

24. Delpisheh A, Brabin L, Attia E, Brabin BJ: Pregnancy late in life: a hospital-based study of birth outcomes. J Womens Health (Larchmt) 2008, I 7:965-970.

25. Gleicher N, Barad D: The relative myth of elective single embryo transfer. Hum Reprod 2006, 2 I: I337-1344.

26. Stern JE, Goldman MB, Hatasaka H, MacKenzie TA, Racowsky C, Surrey ES: Optimizing the number of blastocyst stage embryos to transfer on day 5 or 6 in women 38 years of age and older: a Society for Assisted Reproductive Technology database study. Fertil Steril 2009, 91:157-166.

27. Vestergaard $\mathrm{CH}$, Lidegaard $\mathrm{O}$, Tabor $\mathrm{A}$ : Invasive prenatal diagnostic practice in Denmark 1996 to 2006. Acta Obstet Gynecol Scand 2009, 88:362-365.

28. Fitzsimmons BP, Bebbington MW, Fluker MR: Perinatal and neonatal outcomes in multiple gestations: assisted reproduction versus spontaneous conception. Am J Obstet Gynecol 1998, I79: | |62-I| 67

29. Cervera R, Balasch J: Bidirectional effects on autoimmunity and reproduction. Hum Reprod Update 2008, 14:359-366.

30. Taylor E, Gomel V: The uterus and fertility. Fertil Steril 2008, 89:1-16.

31. Ghobara T, Vandekerckhove P: Cycle regimens for frozenthawed embryo transfer. Cochrane Database Syst Rev 2008:CD0034I4.

\section{Publish with Bio Med Central and every scientist can read your work free of charge}

"BioMed Central will be the most significant development for disseminating the results of biomedical research in our lifetime. "

Sir Paul Nurse, Cancer Research UK

Your research papers will be:

- available free of charge to the entire biomedical community

- peer reviewed and published immediately upon acceptance

- cited in PubMed and archived on PubMed Central

- yours - you keep the copyright

Submit your manuscript here:

http://www.biomedcentral.com/info/publishing_adv.asp
BioMedcentral 